\title{
Seguridad de la ivermectina: toxicidad y reacciones adversas en diversas especies de mamíferos
}

\section{Safety of ivermectin: toxicity and adverse reactions in several mammal species}

\author{
Aránzazu González-Canga, ${ }^{* 1}$ Ph.D, Nélida Fernández-Martínez, ${ }^{1}$ Ph.D, Ana \\ Sahagún-Prieto, ${ }^{1} \mathrm{Ph} . \mathrm{D}$, Juan García-Vieitez, ${ }^{1} \mathrm{Ph} . \mathrm{D}$, María José Díez Liébana, ${ }^{1} \mathrm{Ph} . \mathrm{D}$, \\ Pedro Pablo Tamame-Martín, ${ }^{2}$ M.Sc, Matilde Sierra-Vega, ${ }^{1}$ Ph.D.
}

${ }^{1}$ Universidad de León. Departamento de Ciencias Biomédicas. Área de Farmacología. León. España. ${ }^{2}$ Matadero GYPISA-Talavera. Talavera de la Reina, Toledo. *Correspondencia: agonc@unileon.es

Recibido: Enero 12 de 2010; Aceptado: Julio 8 de 2010.

\section{RESUMEN}

La ivermectina es un fármaco antiparasitario muy utilizado en Medicina Veterinaria, dado su espectro de actividad que abarca tanto endo como ectoparásitos, elevada eficacia y amplio margen de seguridad. No obstante, su administración puede dar lugar a efectos tóxicos. La mayoría de ellos derivan de la sobredosificación del compuesto, aunque también se han descrito, a dosis terapéuticas, casos de susceptibilidad extrema a los efectos neurotóxicos del fármaco en determinadas razas o subpoblaciones de animales, así como reacciones anafilácticas por la destrucción masiva de parásitos.

Palabras clave: ivermectina, toxicidad, efectos adversos.

\section{ABSTRACT}

The antiparasitic drug Ivermectin is frequently used in veterinary medicine, due to its broad spectrum of activity (both against endo- and ectoparasites, high efficacy and wide margin of safety. However, its administration can give rise to toxic effects. Most of them come from the overdosage of the compound, although, at therapeutic doses, it also has been described an increased susceptibility to Ivermectin toxicosis in some breeds and subpopulations of animals, as well as severe anaphylactic reactions due to the sudden massive release of parasite toxins.

Key words: Ivermectin, toxicity, adverse effects. 


\section{NTRODUCCI ÓN}

La ivermectina es un antiparasitario eficaz frente a numerosos nematodos, así como frente a ectoparásitos de diversas especies animales. Este compuesto tiene un amplio margen de seguridad en rumiantes, cerdos y équidos, así como en la mayor parte de las razas de perros. Éste es al menos 10 veces la dosis terapéutica, que oscila entre 6 y $500 \mu \mathrm{g} / \mathrm{kg}(0.006-0.5 \mathrm{mg} / \mathrm{kg})$, según la especie, indicación y vía de administración $(1,2)$. Sin embargo, su administración puede dar lugar a efectos tóxicos. El objetivo del presente trabajo fue resumir las investigaciones realizadas por otros autores en este campo.

Toxicidad aguda. La toxicidad aguda de este compuesto se ha investigado en diversas especies de animales de laboratorio (3). Los signos de toxicidad fueron semejantes tras la administración oral e intraperitoneal, tanto en ratones como en ratas, y consistieron en ataxia, temblores y actividad reducida, muriendo los animales entre 1 y 2 días después. La ivermectina era más tóxica en ratas jóvenes (de 1-2 días) que en adultos, dado el escaso desarrollo de la barrera hematoencefálica, que permitiría el acceso del compuesto al sistema nervioso central, con la consecuente depresión clínica. Los valores de dosis letal, $D L_{50}$, determinados en éste y otros ensayos $(4,5)$ muestran un amplio margen de seguridad del compuesto (Tabla 1 ).

Tabla 1. $\mathrm{DL}_{50}$ de la ivermectina en distintas especies de animales de laboratorio.

\begin{tabular}{cccc}
\hline Autor Especie & $\begin{array}{c}\text { Vía de } \\
\text { administración }\end{array}$ & $\begin{array}{c}\mathbf{D L}_{\mathbf{5 0}} \\
\mathbf{m g} / \mathbf{k g} \text { ) }\end{array}$ \\
\hline (3) & Ratón & oral & 25 \\
& & intraperitoneal & 30 \\
& Rata & oral & 50 \\
& & intraperitoneal & 55 \\
& neonato & oral & $02-$ Mar \\
& & tópica & $>660$ \\
& Conejo & tópica & 406 \\
(4) & Ratón & intraperitoneal & 15 \\
(5) & Rata & subcutánea & 50 \\
\hline
\end{tabular}

Diversos investigadores han relacionado los efectos tóxicos del fármaco con su interacción con la glicoproteína-P, que limitaría su acceso al sistema nervioso central. En este sentido, la ausencia de esta proteína determinaba la acumulación de la ivermectina en el cerebro de ratones transgénicos (alcanzando concentraciones 94 veces más elevadas que en los animales en los que ésta se expresaba con normalidad), lo que coincidía con una mayor susceptibilidad a los efectos neurotóxicos del compuesto en estos ratones $\left(D_{50}\right.$ de 0.6 y $60 \mathrm{mg} / \mathrm{kg}$ ) $(6,7)$. Por otro lado, en ratones CF-1, se atribuyó el que un $25 \%$ de los animales fueran extremadamente susceptibles a estos efectos tóxicos a la ausencia de glicoproteína-P en el epitelio intestinal y el endotelio de los capilares cerebrales, tejidos barrera para el acceso a la circulación sistémica y al sistema nervioso central (8). Así, los niveles plasmáticos y tisulares del fármaco, 24 horas tras su administración oral, fueron mayores en dichos ratones $(2.5 ; 33$; 7 y 2.9 veces, respectivamente, en plasma, cerebro, testículos e intestino delgado, ovarios y vesícula biliar) que en los que no manifestaban signos de toxicidad, en los que, además, se detectaron elevadas cantidades de esta proteína en los tejidos.

Esta susceptibilidad se debería a la expresión alterada, únicamente, del gen mdria. Así, en ratones CF-1 de genotipo mdr1a -/-, y que por tanto no expresaban la proteína en las células endoteliales cerebrales, dosis orales de $0.3-0.8 \mathrm{mg} / \mathrm{kg}$ producían temblores musculares, ataxia y letargia en 2-8 horas, mientras que en ratones con genotipos -/+ y +/+ no se observó neurotoxicidad. Además, todos los animales expresaban con normalidad los genes mdr1b y mdr2 (en glándulas adrenales, riñón y útero gestante: mdr1b, e hígado: mdr2) (9). Asimismo, las concentraciones cerebrales eran mayores en los animales $-/-$, y se incrementaban a medida que trascurría el tiempo de la administración, alcanzando a las 24 horas valores 70 veces superiores a los de ratones $+/+$, en los que los niveles se habían mantenido estables (10).

En el ganado vacuno se observó que una dosis subcutánea $(8 \mathrm{mg} / \mathrm{kg}$ de ivermectina 
formulada en propilenglicol) producía ataxia, fasciculaciones musculares, depresión motora, decúbito lateral, bradicardia y midriasis, muriendo uno de los siete animales tratados. Signos idénticos fueron descritos en animales que recibieron únicamente el vehículo, atribuyéndose, por tanto, el efecto tóxico al vehículo de la formulación (11).

De forma similar, también se atribuyó al vehículo la toxicidad, en ovejas que ingirieron ivermectina ( $8 \mathrm{mg} / \mathrm{kg}$ ) formulada en propilenglicol y que presentaron ataxia seguida de postración, dado que los animales que recibieron sólo el propilenglicol manifestaron los mismos signos y que no apareció signo alguno en ovejas tratadas con una formulación acuosa (12).

Por su parte, ni en cabras (13) ni renos (14) se han observado signos de toxicidad con dosis subcutáneas de 1.6 ó $2 \mathrm{mg} / \mathrm{kg}$, respectivamente.

En el cerdo, una dosis subcutánea de 30 $\mathrm{mg} / \mathrm{kg}$ daba lugar, en 24 horas, a ataxia y letargia, midriasis, postración y anorexia, mientras que con $15 \mathrm{mg} / \mathrm{kg}$ no se produjeron signos de toxicidad (15).

Signos parecidos a los anteriormente citados, se describieron, tras administrar una dosis oral de $2 \mathrm{mg} / \mathrm{kg}$, en caballos (durante dos días consecutivos) (16) y en una cebra de 3 meses (17): ataxia, depresión y pérdida parcial de la visión, recuperándose completamente todos los animales. Recientemente, aunque en este caso a la dosis oral de $0.2 \mathrm{mg} / \mathrm{kg}$, tres caballos mostraron signos de intoxicación severa al compuesto. Dos de los animales se recuperaron y el tercero fue sacrificado, detectándose elevadas concentraciones de ivermectina en el tejido cerebral (18).

La sobredosificación y la susceptibilidad en función de la raza son las dos causas de toxicidad en perros. Así, dosis orales de $10 \mathrm{mg} / \mathrm{kg}$ daban lugar, en Beagles, a midriasis, ataxia, temblores musculares y muerte en 12-24 horas, calculándose una $\mathrm{DL}_{50}$ de $80 \mathrm{mg} / \mathrm{kg}$ (3). Otros autores (19) encontraron signos semejantes en un Doberman Pinscher que ingirió $3.5 \mathrm{mg} / \mathrm{kg}$ : temblores, decúbito lateral y midriasis.
En perros de raza Collie se han observado casos de intoxicación caracterizados por la aparición de signos que reflejan la afectación del sistema nervioso central. Así, administrando dosis orales de $0.1-2.5 \mathrm{mg} / \mathrm{kg}$, se comprobó que, con $0.1 \mathrm{mg} / \mathrm{kg}$ tres de los catorce perros utilizados desarrollaban signos medios de toxicidad (sialorrea, vómitos, temblores y ataxia), mientras que con $0.2 \mathrm{mg} / \mathrm{kg}$ se producían signos severos (temblores que progresaban a debilidad, depresión y coma) en el $50 \%$ de los animales. A las dosis de 0.6 y $2.5 \mathrm{mg} / \mathrm{kg}$, sólo ensayadas en perros que no habían mostrado signos severos previamente, únicamente en uno de ellos hubo manifestaciones de toxicidad. Los autores clasificaron, por tanto, a los animales como sensibles e insensibles, al manifestar o no signos de toxicidad con la dosis de $0.2 \mathrm{mg} /$ kg (20).

Inicialmente se descartó que, en esta raza, la toxicidad surgiera de niveles plasmáticos más elevados en los perros sensibles (21) ya que, con una dosis oral de $0.1 \mathrm{mg} / \mathrm{kg}$, la concentración plasmática máxima, el tiempo al que ésta se alcanza y el área bajo la curva de niveles plasmáticos eran semejantes en dos lotes de animales: sensibles e insensibles, según la clasificación propuesta por Paul et al (20) También se descartó que se debiera a una mayor concentración de ivermectina libre, al no detectarse diferencias significativas en los porcentajes de fármaco libre - unido a las proteínas plasmáticas en Collies sensibles e insensibles (22). Estos autores consideraron que estas diferencias podían deberse a otro aspecto de la distribución del fármaco, en concreto a un paso diferente de la barrera hematoencefálica, cuyo origen podían ser diferencias anatómicas o fisiológicas. Una hipótesis semejante había sido propuesta previamente por Pulliam et al (23), que detectaron, con una dosis oral de $0.2 \mathrm{mg} / \mathrm{kg}$, mayor cantidad de ivermectina (entre 10 y 100 veces) en el cerebro de aquellos Collie que habían mostrado signos neurológicos, sugiriendo una mayor permeabilidad de la barrera hematoencefálica en estos animales.

Recientemente, se ha atribuido la susceptibilidad extrema de algunos Collies a 
que carecen de glicoproteína $P(24,25)$. Estos autores detectaron una mutación a nivel del cuarto exón del gen que codifica dicha proteína, que da lugar a un defecto en su traducción. Así, la secuencia de este gen se correspondía en un $99.9 \%$ a la de perros no susceptibles al fármaco, situándose la única diferencia en la ausencia, en los Collies susceptibles, de cuatro pares de bases en el ADN. Este cambio estructural resulta en la traducción, en los animales homocigóticos, de un codón stop prematuro, obteniéndose así una proteína de sólo un $7.1 \%$ de la longitud normal.

En este sentido, ya se han realizado estudios para determinar el porcentaje de Collies que portan el alelo mutante. Así, en Estados Unidos (26) se comprobó que de los 40 animales estudiados, un 35\% (14 perros) eran homocigóticos para el alelo mutante, mientras que un $42 \%$ ( 17 animales) eran heterocigóticos, concluyendo que un elevado porcentaje de Collies podría presentar reacciones adversas a la ivermectina. En Francia (27), los porcentajes hallados para animales homocigóticos y heterocigóticos fueron, respectivamente, 48 y $32 \%$, de un total de 83 Collies.

Aunque los trabajos más numerosos se han llevado a cabo en Collies, también se ha descrito hipersusceptibilidad en otras razas, fundamentalmente perros de pastor. Así, un Bobtail que ingirió $0.15 \mathrm{mg} / \mathrm{kg}$ presentó, entre otros signos, decúbito lateral, midriasis y nistagmo (28), mientras que en dos Pastores Australianos tratados con $0.15-0.3 \mathrm{mg} / \mathrm{kg}$ se observó sialorrea, hipotermia, bradicardia, bradipnea, ausencia de reflejo pupilar y coma (29).

En este sentido, varios autores han determinado, en diversos países, la frecuencia del alelo mutante en diferentes razas de perros de pastor (30-32), destacando la elevada proporción de animales que presentaba esta mutación y la importancia de su conocimiento a la hora de establecer un tratamiento farmacológico en estas razas, por la posibilidad de presentación de reacciones adversas graves. Así, en Australia se estudió (32) la frecuencia del alelo mutante en las siguientes razas:
Collie (33 animales), Pastor Australiano (17 animales), Border Collie (7 animales) y Shetland ( 7 animales). Los resultados obtenidos por estos autores se muestran en la tabla 2.

Tabla 2. Genotipos para el gen MDR1 en distintas razas de perros en Australia.

\begin{tabular}{lccc}
\hline \multicolumn{1}{c}{ RAZA } & $\begin{array}{c}\text { GENOTIPO (número de animales) } \\
+/+\end{array}$ & $+/-$ & $-/-$ \\
\hline Collie & 4 & 21 & 8 \\
$\begin{array}{l}\text { Pastor } \\
\text { Australiano }\end{array}$ & 5 & 6 & 3 \\
Border Collie & 7 & 0 & 0 \\
Shetland & 4 & 3 & 0 \\
\hline
\end{tabular}

En un estudio realizado el mismo año en Alemania (30), en el que se usó un número mucho más elevado de animales que en los trabajos realizados hasta el momento, se obtuvieron resultados similares. Éstos se detallan a continuación, en la tabla 3.

Tabla 3. Genotipos para el gen MDR1 en distintas razas de perros en Alemania.

\begin{tabular}{|c|c|c|c|c|}
\hline \multirow{2}{*}{ RAZA } & \multicolumn{3}{|c|}{ GENOTIPO (\%) } & \multirow{2}{*}{$\begin{array}{c}\text { Número de } \\
\text { animales }\end{array}$} \\
\hline & $+/+$ & $+/-$ & $-/-$ & \\
\hline Collie & 23.9 & 43.1 & 33 & 578 \\
\hline Shetland & 45.7 & 48.6 & 5.7 & 140 \\
\hline $\begin{array}{l}\text { Pastor } \\
\text { Australiano }\end{array}$ & 67.9 & 25.2 & 6.9 & 333 \\
\hline Border Collie & 99.1 & 0.6 & 0 & 334 \\
\hline Bobtail & 87.5 & 12.5 & 0 & 24 \\
\hline Waller & 62.9 & 37.1 & 0 & 62 \\
\hline
\end{tabular}

Otro trabajo, desarrollado en Japón (31), estudió la frecuencia de esta mutación en ocho razas de perros. Estos autores, además del Collie, Pastor Australiano y Shetland, ampliaron las razas de perros investigadas, incluyendo grupos diferentes al de perros de pastor: Golden Retriever y Labrador Retriever (perros cobradores de caza), Dachshund (Teckels) y 2 razas de origen japonés: Shih Tzu (perros de compañía) y Shiba Inu (perros de trineo). Los porcentajes de animales que presentaban el alelo mutante fue muy elevado en el Collie (58.3\% de 12 animales) y el Pastor Australiano (33.3\% de 9 animales), siendo de $1.2 \%$ en el Shetland 
González-Canga - Seguridad de la ivermectina: toxicidad y reaciones adversas 2133

(42 animales). En el resto de las razas ensayadas no se encontró este alelo.

Por otra parte, en un estudio desarrollado en Estados Unidos e Inglaterra (33), se obtuvieron los siguientes resultados (Tabla 4).

Tabla 4. Genotipos para el gen MDR1 en distintas razas de perros en Estados Unidos e Inglaterra.

\begin{tabular}{lcccc}
\hline \multicolumn{1}{c}{ RAZA } & \multicolumn{3}{c}{ GENOTIPO (\%) } & $\begin{array}{c}\text { Número de } \\
\text { animales }\end{array}$ \\
\hline Collie & 22 & 46.8 & 31.2 & 263 \\
Shetland & 84.2 & 14.7 & 1.1 & 190 \\
Pastor Australiano & 68.5 & 29.8 & 1.7 & 178 \\
$\begin{array}{l}\text { Pastor Australiano } \\
\text { (miniatura) }\end{array}$ & 51.8 & 44.6 & 3.6 & 56 \\
Bobtail & 92.7 & 7.3 & 0 & 151 \\
McNab & 68.6 & 28.6 & 2.8 & 35 \\
Whippet & 32.6 & 51.7 & 15.7 & 89 \\
Silken Windhound & 65.5 & 33.3 & 1.2 & 84 \\
\hline
\end{tabular}

Si bien los porcentajes calculados son semejantes a los determinados en estudios previos, estos investigadores resaltaron el hecho de haber hallado el gen mutante en las dos razas de perros no pastores que emplearon en su ensayo: Whippet y Silken Windhound (lebreles).

No obstante, hay que tener en cuenta que, en esta especie, el margen de seguridad es amplio ya que la ivermectina se administra mensualmente a la dosis de $0.006 \mathrm{mg} / \mathrm{kg}$, dosis basada en la eficacia frente a Dirofilaria immitis (34) y muy alejada de las anteriormente señaladas.

En cuanto a los gatos, una dosis subcutánea de $0.3 \mathrm{mg} / \mathrm{kg}$ causó miosis, ataxia, temblores musculares, debilidad, coma y la muerte, a un animal de 4 meses (35). Signos similares se describieron en dos gatos de un mes, pero tratados con dosis 250 y 125 veces superiores a la terapéutica, así como en un gato de 2 años que recibió unas 16 veces la dosis terapéutica por vía subcutánea: salivación, lacrimeo, midriasis, taquipnea, taquicardia y ataxia (36).
Toxicidad subaguda y crónica. En animales de laboratorio, la ingestión diaria de ivermectina durante el período de organogénesis en ratones $(0.4-0.8 \mathrm{mg} / \mathrm{kg})$, ratas $(10 \mathrm{mg} / \mathrm{kg})$ y conejos $(3-6 \mathrm{mg} / \mathrm{kg})$, incrementó la incidencia de paladar hendido. No obstante, no se la consideró embriotóxica dado que la frecuencia de presentación de anomalías era muy baja (3). En otro estudio (37), la incidencia de paladar hendido determinada tras la administración oral diaria de $1.5 \mathrm{mg} / \mathrm{kg}$, durante 9 días, a ratones CF- 1 hembras, de un derivado de la ivermectina, fue de $100 ; 41$ y $0 \%$, respectivamente, en fetos con genotipos mdr1a -/-, +/- y +/+, lo que reflejaría la importancia de que la glicoproteína-P forme parte de la barrera fetoplacentaria, a la hora de proteger al feto de potenciales teratógenos.

En ratas preñadas tratadas diariamente, a lo largo de 70 días, con dosis orales de 0.05; $0.1 ; 0.2 ; 0.4 ; 1.2$ y $3.6 \mathrm{mg} / \mathrm{kg}$, se comprobó que a partir de $0.4 \mathrm{mg} / \mathrm{kg}$ aumentaba la mortalidad de las crías de hasta 10 días de edad (coincidiendo con el periodo de formación de la barrera hematoencefálica ) y que descendía el peso de los supervivientes en relación con los neonatos de madres no tratadas. Y se comprobó que esta toxicidad neonatal se producía a través de la leche y no por la exposición en el útero (38).

Recientemente, se ha evaluado el efecto de la ivermectina sobre la fertilidad de ratas macho, administrándose, para ello, intraperitonealmente, durante 8 semanas a la dosis semanal de $0.3 \mathrm{mg} / \mathrm{kg}$ (39). El compuesto dio lugar a un ligero descenso en el peso de los testículos, epidídimo, vesículas seminales y próstata, si bien no influía ni en la histomorfología de dichos órganos ni en las características del semen. La coadministración con verapamil, también sustrato de la glicoproteína-P, potenció este efecto $y$, además, provocó anomalías histomorfológicas y alteró el semen (disminuyendo la motilidad de los espermatozoides y causando alteraciones en su morfología), lo que se explicó en base a una interacción entre ambos compuestos que habría facilitado el paso de la ivermectina a través de la barrera hematotesticular. 
También en conejos macho se determinó que la administración repetida de la ivermectina originaba una disminución en el peso de los órganos sexuales (40).

La ivermectina no tuvo repercusiones negativas sobre la implantación ni el desarrollo embrionario y fetal, tras su administración a una dosis del doble de la terapéutica, repetida bien durante la primera fase de la gestación bien en el periodo final de la preñez, por vía subcutánea a vacas (11), ovejas (12) y cerdas (41), y por vía oral a yeguas (42), siendo la descendencia normal en todos los casos, al igual que en perros Beagle que ingirieron dosis dobles de la terapéutica, a intervalos mensuales, durante un total de 8 meses (43).

El antiparasitario tampoco alteró el potencial reproductivo de los machos. La administración subcutánea del doble de la dosis terapéutica no redujo la calidad del semen en toros (11) o cerdos (41), ni afectó a la libido, el peso o la histomorfología de los testículos en la primera especie (11). En cuanto a la especie ovina, se indicó que la ivermectina reducía la concentración de espermatozoides y su motilidad (44). Esto contrasta con lo señalado en un ensayo previo (45), en el que con una dosis oral del doble de la terapéutica, repetida 6 veces a intervalos de 21 días, no hubo cambios ni en la histología testicular ni en la calidad del semen en machos de raza Merina, de forma similar a lo comprobado en caballos sementales tratados por vía oral a la dosis habitual (46).

Finalmente, en monos rhesus adultos que ingirieron, diariamente durante 16 días, 1.2 $\mathrm{mg} / \mathrm{kg}$ no se detectaron efectos indeseables, ni tampoco en neonatos que recibieron, durante el mismo tiempo, dosis diarias de $0.1 \mathrm{mg} / \mathrm{kg}$, animales en los que no se encontraron ni anomalías en los parámetros hematológicos o bioquímicos ni en la anatomía e histología de los distintos órganos (3).
Reacciones adversas. En el ganado vacuno, el tratamiento con ivermectina en animales infectados por Hypoderma bovis e H. lineatum, provocó, respectivamente, hemorragias en el canal espinal y paresia, así como esofagitis edematosa, relacionándose dichas reacciones adversas con la muerte de las larvas provocada por el fármaco (47). La incidencia de estas reacciones es muy baja, de un $0.0001 \%$, y se evitaría no tratando a los animales durante la migración larvaria (1).

También en los caballos se ha descrito la aparición de edema subcutáneo, sobre todo en la línea ventral media, que se atribuyó a una reacción de hipersensibilidad a las microfilarias muertas de Onchocerca cervicalis al administrar la ivermectina, resolviéndose el edema en 3-4 días sin terapia adicional (48). En 1984, Karns y Luther (49) señalaron que 366 de 3316 animales desarrollaban reacciones adversas, consistiendo el $91 \%$ de ellas en edema de la línea ventral media.

En esta especie, se observaron importantes efectos adversos tras la administración intramuscular de ivermectina, que fueron achacados a la inyección intravenosa accidental o a una hipersensibilidad al excipiente. Se manifestaba la toxicidad con graves reacciones de tipo anafiláctico, que podían llegar a ser letales, lo que obligó a retirar la formulación intramuscular del mercado estadounidense 17 meses después de su introducción $(15,50,51)$.

En conclusión, aunque la ivermectina es, en líneas generales, un fármaco seguro y bien tolerado, su administración puede dar lugar a efectos tóxicos, bien como consecuencia de la sobredosificación, bien por hipersusceptibilidad al compuesto. Dado que no hay antídoto específico y las alternativas se reducen al tratamiento sintomático, es importante conocer los posibles efectos tóxicos derivados de su uso y, más aún, cuando se emplea en especies distintas, o para indicaciones diferentes, a aquellas en las que está autorizada . 


\section{REFERENCI AS}

1. McKellar QA, Benchaoui HA. Avermectins and milbemycins. J Vet Pharmacol Ther 1996; 19: 331-351.

2. Burkhart CN. Ivermectin: an assessment of its Pharmacology, Microbiology and safety. Vet Hum Toxicol 2000; 42: 3035.

3. Lankas GR, Gordon LR. Toxicology. En: Campbell WC, ed. Ivermectin and abamectin. New York: Springer-Verlag; 1989: 89-112.

4. Marques-Santos LF, Bernardo RR, Paula E, Rumjanek VM. Cyclosporin A and trifluoperazine, two resistancemodulating agents, increase ivermectin neurotoxicity in mice. Pharmacol Toxicol 1999; 84: 125-129.

5. Dadarkar SS, Deore MD, Gatne MM. Comparative evaluation of acute toxicity of ivermectin by two methods after single subcutaneous administration in rats. Regul Toxicol Pharmacol 2007; 47: 257-260.

6. Schinkel AH, Smit JJM, Van Telligen O, Beijnen JH, Wagenaar E, Van Deemter $L$, et al. Disruption of the mouse mdr1a P-glycoprotein gene leads to a deficiency in the blood brain barrier and to increased sensivity to drugs. Cell 1994; 77: 491-502.

7. Schinkel $A H$, Wagenaar $E$, Van Deemter $L$, Mol CA, Borst P. Absence of mdr1a $\mathrm{P}$-glycoprotein in mice affects tissue distribution and pharmacokinetics of dexamethason, digoxin and ciclosporyn A. J Clin Invest 1995; 96: 1698-1705.

8. Lankas GR, Cartwright ME, Umbenhauer DR. P-glycoprotein deficiency in a subpopulation of mice enhances avermectin-induced neurotoxicity. Toxicol Appl Pharmacol 1997; 143: 357365.
9. Umbenhauer DR, Lankas GR, Pippert TR, Wise LD, Cartwright ME, Hall SJ, et al. Identification of a P-glycoproteindeficient subpopulation in the CF-1 mouse strain using a restriction fragment length polymorphism. Tox Appl Pharm 1997; 146: 88-94.

10. Kwei GY, Alvaro RF, Chen Q, Jenkins HJ, Hop CE, Keohane CA, et al. Disposition of ivermectin and cyclosporin A in CF-1 mice deficient in mdr1a P-glycoprotein. Drug Metab Dispos 1999; 27: 581-587.

11. Leaning WHD, Roncalli RA, Brokken ES. The efficacy and safety evaluation of ivermectin: a new injectable antiparasitic agent for cattle. En: Leaning W, Siegmund O, Fraser C, eds. Recent developments in the control of animal parasites. Proceedings of the MSD AGVET Symposium at the XXII World Veterinary Congress. Perth: MSD AGVET; 1983: 25-41.

12. Hotson IK. The development of ivermectin as an antiparasitic agent in sheep. En: Leaning W, Siegmund O, Fraser C, eds. Recent developments in the control of animal parasites. Proceedings of the MSD AGVET Symposium at the XXII World Veterinary Congress. Perth: MSD AGVET; 1983: 42-48.

13. Njanja JC, Bell JF, Wescott RB. Apparent lack of toxicity in adult East African goats on parenterally administered ivermectin. Bull Anim Health Prod Afr 1985; 33: 123-127.

14. Dieterich RA, Craigmill AL. Safety, efficacy, and tissues residues of ivermectin in reindeer. Rangifer 1990; 10: 53-56. 
15. Pulliam JD, Preston JM. Safety of ivermectin in target animals. En: Campbell WC, ed. Ivermectin and abamectin. New York: Springer-Verlag; 1989: 149-161.

16. Leaning WHD. The efficacy and safety evaluation of ivermectin as a parenteral and oral antiparasitic agent in horses. En: Milne FJ, ed. Proceedings of the $29^{\text {th }}$ Annual Convention of the American Association of Equine Practitioners. Las Vegas: American Association of Equine Practitioners; 1983: 319-328.

17. Hautekeete LA, Khan SA, Hales WS. Ivermectin toxicosis in a zebra. Vet Human Toxicol 1998; 40: 29-31.

18. Swor TM, Whittenburg JL, Chaffin MK. Ivermectin toxicosis in three adult horses. J Am Vet Med Assoc 2009; 235: 558-562.

19. Hopkins KD, Marcella KL, Strecker AE. Ivermectin toxicosis in a dog. J Am Vet Med Assoc 1990; 197: 93-94.

20. Paul AJ, Tranquilli WJ, Seward RL, Todd KS, DiPietro JA. Clinical observations in Collies given ivermectin orally. Am J Vet Res 1987; 48: 684-685.

21. Tranquilli WJ, Paul AJ, Seward RL. Ivermectin plasma concentration in Collies susceptible to ivermectin induced toxicosis. Am J Vet Res 1989; 50: 769770.

22. Rohrer SP, Evans DV. Binding characteristics of ivermectin in plasma from Collie dogs. Vet Res Commun 1990; 14: 157-165.

23. Pulliam JD, Seward RL, Henry RT, Steinberg SA. Investigating ivermectin toxicity in Collies. Vet Med 1985; 80: 33-40.

24. Mealey KL, Bentjen SA, Gay JM, Cantor $\mathrm{GH}$. Ivermectin sensitivity in Collies is associated with a deletion mutation of the mdr1 gene. Pharmacogenetics 2001; 11: 727-733.
25. Roulet A, Puel O, Gesta S, Lepage JF, Drag $M$, Soll $M$, et al. MDR1-deficient genotype in Collie dogs hypersensitive to the P-glycoprotein substrate ivermectin. Eur J Pharmacol 2003; 460: 85-91.

26. Mealey $\mathrm{KL}$, Bentjen SA, Waiting DK. Frequency of the mutant MDR1 allele associated with ivermectin sensitivity in a sample population of Collies from the Northwestern United States. Am J Vet Res 2002; 63: 479-481.

27. Hugnet $\mathrm{C}$, Bentjen SA, Mealey $\mathrm{KL}$. Frequency of the mutant MDR1 allele associated with multidrug sensitivity in a sample of Collies from France. J Vet Pharmacol Ther 2004; 27: 227-229.

28. Houston DM, Parent J, Matushek KJ. Ivermectin toxicosis in a dog. J Am Vet Med Assoc 1987; 191: 78-80.

29. Hadrick MK, Bunch SE, Kornegay JN. Ivermectin toxicosis in two Australian shepherds. J Am Vet Med Assoc 1995; 206: 1147-1152.

30. Geyer J, Döring B, Godoy JR, Leidolf R, Moritz $A$, Petzinger $E$. Frequency of the nt230 (del4) MDR1 mutation in Collies and related dog breeds in Germany. J Vet Pharmacol Ther 2005; 28: 545-551.

31. Kawabata A, Momoi Y, Inoue-Murayama M, Iwasaki T. Canine MDR1 gene mutation in Japan. J Vet Med Sci 2005; 67: 1103-1107.

32. Mealey $\mathrm{KL}$, Munyard KA, Bentjen SA. Frequency of the mutant MDR1 allele associated with multidrug sensitivity in a sample of herding breed dogs living in Australia. Vet Parasitol 2005; 131: 193196.

33. Neff MW, Robertson KR, Wong AK, Safra $\mathrm{N}$, Broman KW, Slatkin M, et al. Breed distribution and history of canine MDR11 Delta, a pharmacogenetic mutation that marks the emergence of breeds from the collie lineage. Proc Natl Acad Sci USA 2004; 101: 11725-11730. 
34. Campbell WC. Use of ivermectin in dogs and cats. En: Campbell WC, ed. Ivermectin and abamectin. New York: Springer-Verlag; 1989: 245-259.

35. Lewis DT, Merchant SR, Neer TM. Ivermectin toxicosis in a kitten. J Am Vet Med Assoc 1994; 205: 584-586.

36. Muhammad G, Abdul J, Khan MZ, Saqib $M$. Use of neostigmine in massive ivermectin toxicity in cats. Vet Hum Toxicol 2004; 46: 28-29.

37. Lankas GR, Wise LD, Cartwright ME, Pippert T, Umbenhauer DR. Placental Pglycoprotein deficiency enhances susceptibility to chemically induced birth defects in mice. Reprod Toxicol 1998; 12: 457-463.

38. Lankas GR, Minsker DH, Robertson RT. Effects of ivermectin on reproduction and neonatal toxicity in rats. Food Chem Toxicol 1989; 27: 523-529.

39. El-Nahas AF, El-Ashmawy IM. Effect of ivermectin on male fertility and its interaction with P-glicoproteína inhibitor (verapamil) in rats. Environ Toxicol Pharmacol 2008; 26 : 206-211.

40. El-Ashmawy IM, Mandour AA. Studies on the influence of ivermectin on reproductive organs and liver function in male rabbits. En: Third Vet Med Conf. Zagazig: Egypt; 1996.

41. Brokken ES, Barth D, Foster AG, Pulliam JD, Wallace DH. Ivermectin: a new broad-spectrum antiparasitic agent for swine. En: Leaning W, Siegmund O, Fraser C, eds. Recent developments in the control of animal parasites. Proceedings of the MSD AGVET Symposium at the XXII World Veterinary Congress. Perth: MSD AGVET; 1983: 239-258.

42. McKissick GE, Sutherland IH, Foix J, Olson G. The safety of ivermectin administered orally to pregnant mares. Equine Vet J 1987; 7: 357-367.
43. Daurio CP, Gilman MR, Pulliam JD, Seward $\mathrm{RL}$. Reproductive evaluation of male Beagle dogs and the safety of ivermectin. Am J Vet Res 1987; 48: 1755-1760.

44. Tanyildizi, S, Bozkurt T. An investigation of the effects of ivermectin on blood serum, semen hyaluronidase activities and spermatological characteristics in sheep. Turk J Vet Anim Sci 2002; 26: 353-357.

45. Schröder J, Swan GE, Barrick RA, Pulliam JD. Effect of ivermectin on the reproductive potential of breeding rams. J S Afr Vet Assoc 1986; 57: 211-213.

46. Janett $F$, Thun $R$, Ryhiner A, Burger $D$, Hassig $M$, Hertzberg $H$. Influence of Eqvalan ${ }^{\circledR}$ (ivermectin) on quality and freezability of stallion semen. Theriogenology 2001; 55: 785-792.

47. Campbell WC, Benz GW. Ivermectin: a review of efficacy and safety. J Vet Pharmacol Ther 1984; 7: 1-16.

48. Herd RP, Donham JC. Efficacy of ivermectin against Onchocerca cervicalis microfilarial dermatitis in horses. Am J Vet Res 1983; 44: 1102-1105.

49. Karns PA, Luther DG. A survey of adverse effects associated with ivermectin use in Louisiana horses. J Am Vet Med Assoc 1984; 185: 782-783.

50. Campbell WC, Leaning WHD, Seward RL. Use of ivermectin in horses. En: Campbell WC, ed. Ivermectin and abamectin. New York: Springer-Verlag; 1989: 234-244.

51. Breukink HJ, Eysker M. Intravenous administration of Ivomec $^{\circledR}$ in horses. Tijdschr Diergeneeskd 1995; 120: 113114. 